\title{
The Human Genome Project and medical genetics
}

The coordinated efforts now in progress across the world to map and characterise the human genome have, until recently, seemed remote from the immediate problems and challenges of medical genetics. A decade ago the possibility of an overall genome project had scarcely been considered, and while the idea of using DNA polymorphisms to produce a framework map had been raised, ${ }^{1}$ the gene mapping community was composed primarily of human geneticists involved with blood groups and protein polymorphisms. Five years ago, molecular geneticists had rapidly come to dominate the gene mapping scene, as reflected in the successive reports of the Human Gene Mapping Workshops, ${ }^{2}$ but the early successes of isolating human disease genes were more related to efforts directed at the individual genes rather than to the gene map itself.

Since then the avalanche of disease genes mapped, together with the growing number actually cloned, has led to a situation where the majority of serious human genetic disorders are now mapped with sufficient accuracy to allow prediction within families, while for some, such as cystic fibrosis, Duchenne muscular dystrophy, and neurofibromatosis, a detailed exploration of molecular pathology is becoming possible. The power of the new microsatellite markers ${ }^{3}$ and the relative simplicity of PCR techniques have meant that gene mapping research is no longer the preserve of the sophisticated basic molecular laboratory, but can be carried out in many centres closely linked with medical genetics services.

This shift in centre of gravity has been reflected in the general interest now taken in human gene mapping by medical geneticists, both clinicians and those scientists involved primarily with molecular diagnostic laboratories. It is now a topic of immediate clinical interest and application, rather than a distant prospect for the future. Yet this change does not seem to have been fully appreciated by the growing number of organisations and committees involved, whose primary focus is still on the laboratory and computing technology required to produce a detailed and complete map and sequence of the human genome.

As regards the various genome projects initiated in the United States, the European Community, Japan, and Britain, the medical genetics community has had relatively little input to the planning of research programmes, to the ordering of priorities, or to the format and scope of meetings. Individual research projects related to mapping of specific diseases and based in clinical centres have not, in general, been favoured as regards funding.

Thus in a journal devoted primarily to medical genetics, it is worth re-emphasising the central role of medical geneticists as active and essential participants, rather than as passive recipients of new developments. Careful clinical assessment is obviously vital for the detection or exclusion of heterogeneity, for the accurate assessment of rare recombinants, and for correlation of individual mutations with specific phenotypes. The molecular diagnostic laboratory is likely to be a valuable resource of unrelated small families for mapping by linkage disequilibrium, and the increasing use of DNA banks provides the main source of samples for rare genetic disorders. Accurate clinical and cytogenetic descriptions involving deletions or translocations have located a series of important genes, familial polyposis coli on $5 \mathrm{q}$ being a notable example. ${ }^{4}$

This important medical role is not confined to the identification of disease genes, but has been a major stimulus to the development of the overall gene map. A glance at how this has progressed shows a strong correlation between a well developed map of a region and the presence of an important disease. The detailed mapping of $4 p$ and $7 q$ around the Huntington's disease and cystic fibrosis loci are obvious examples, while distal $4 \mathrm{q}$ was almost unknown territory until the facioscapulohumeral dystrophy gene was located there and rapidly stimulated a considerable map around it. It could be argued that, rather than the medical field being the long term recipient of advances from the various genome projects, the converse is true, that workers on genetic disorders, and the medical charities funding them, have been largely responsible for much of the human gene map that has been produced so far. In fact neither view is the entire truth, medical geneticists and basic scientists both benefitting from each other's contribution, but the medical contribution certainly deserves greater notice than it has received.

A further area of medical importance arising from the emerging human gene map concerns some of the ethical implications. There has been a tendency to be dismissive of these in some quarters ${ }^{5}$ (an attitude that is likely to reflect ignorance as well as an understandable irritation at the growing number of professional bioethicists), but the major genome projects, including those of the United States and European Community, have wisely, from the beginning, placed considerable emphasis on studying these. Workers in medical genetics are already meeting some of these issues in practice, such as the use of molecular tests for insurance or legal purposes, ${ }^{6}$ the testing of children for late onset disorders, ${ }^{7}$ and the broad area of privacy and confidentiality. Many clinicians outside genetics have little awareness of these issues, so that medical geneticists, whether clinicians or scientists, are currently the people who have to recognise and avoid such problems arising in the context of genetic testing.

A final area of medical relevance that has not received the attention it deserves is the free availability of DNA probes for diagnostic use. Many of us feared in the early stages that commercial interests would place major restrictions on this, and that biotechnology companies would assume a dominant position that would impede the equitable delivery of health care in relation to molecular testing for genetic diseases. In general this has not happened, something that reflects well on the scientific community. It would be difficult to think of another area of medicine where important advances have been made available so rapidly and widely across the world. This is already an achievement that the Human Genome Projects can be proud of. The various resource centres set up for distributing probes, 
establishing cell lines, and disseminating information have all contributed to this positive outcome.

How will the situation change as the human gene map approaches completion and we gain a more detailed idea of the structure and function of the numerous genes involved in disease susceptibility as well as those for rare mendelian disorders? The medical implications of this are already becoming clear to clinicians in all fields, so the medical geneticist is likely to become the central figure in how these new tools can be and should be used, in education regarding the issues involved, as well as in practical applications for a large body of genetic disorders.

Increasing application of molecular approaches for population screening and the emergence of gene therapy as a reality will further broaden the clinical foundations of this field. When the time comes for the basic scientists involved to move on to other challenges, the medical genetics profession will be left as the principal custodians of this, the most valuable, but potentially the most dangerous, resource of our time.

PETER S HARPER

Institute of Medical Genetics,

University of Wales College of Medicine,

Heath Park,

Cardiff CF4 $4 X N$.

1 Botstein D, White RL, Skolnick M, Davies RW. Construction of a genetic linkage map in Man using restriction length fragment polymorphisms. $A m$ f Hum Genet 1980;32:314-31.

2 Human Gene Mapping International Workshop Reports. Cytogenet Cel Genet 1987-1991 (supplements).

3 Weber JL, May PE. Abundant class of human DNA polymorphisms which can be typed using the polymerase chain reaction. Am f Hum Genet 1989;44:388-96.

4 Bodmer WF, Bailey CJ, Bodmer J, et al. Localisation of the gene for familial adenomatosis polyposis on chromosome 5 . Nature 1987;328:614-6.

5 Editorial. Ethical problems to merit the name. Nature 1991;352:359-60.

6 Pokorski R. Genetic testing in insurance. Bridging the gap between new knowledge and its application. Contingencies September-October 1990: know.

7 Harper PS, Clarke A. Should we test children for 'adult' genetic disease. Lancet 1990;335:1205-6. 